\title{
A Game Controller Based on Multiple Sensors
}

\author{
Dapeng Zhang, Zhongjie Cai, Kefei Chen, Bernhard Nebel \\ Research Group on Foundations of Artificial Intelligence \\ Department of Information \\ Universtiy of Freiburg \\ zhangd, caiz, chenk, nebel@informatik.uni-freiburg.de
}

\begin{abstract}
A digital game is normally controlled by hand. Playing such a game requires only minimum hand movements. Rather than being easy and comfortable, this game controller is designed to be physically taxing for the players. It consists of several sensors, which makes a game more lively and forces the users to be more physically active. By using different mapping methods, one game can be played in several ways. The statistics gathered from the experiments show that even though the quality of control on the chosen fighting game is not as high as with a normal joystick, the developed controller is still preferred by most of the participants. It induces much more movement than a normal joystick.
\end{abstract}

\section{Keywords}

Human-Computer Interaction, Game Controller

\section{INTRODUCTION}

There are many fantastic digital games. However, playing such a game is regarded as an unhealthy activity [2]. The main reason is that most of the games can be fully controlled by hand. It would be unhealthy if children stayed on the couch for several hours, gazing at the screen and moving only their fingers.

"More Movement" is a popular idea for playing digital games. This concept can be found in current commercial-available game consoles. For example, accelerometers are integrated into the remote controller of $w i i^{1}$, which requires players to twist their wrists. A camera (EyeToy and ${ }^{2} \mathrm{Natal}^{3}$ ) is used to recognize the gestures of a player, so that one needs to wave their arms to control a game. Although these devices introduce "more movements" than a normal joystick does, their effects are still limited.

${ }^{1}$ wii is a product of Nintendo

${ }^{2}$ EyeToy is a product of Sony

${ }^{3} \mathrm{Natal}$ is the future concept for the game console of Microsoft
The main idea of the work here is to enable the players to control the game by their activities, which involve not only hands and feet, but also the movements of the whole body. In addition, there could be other inputs such as the voice. Different from the idea of a single device, e.g. joystick, the proposed game controller can have multiple parts (sensors) scattered at different places in the environment. The controller can be used not only for a single player, but also for a team of players cooperating on a task. It raises the question on how to configure the mapping, which is from real-world activities to a sequence of commands to control a game. Some algorithms in artificial intelligence, such as finite state machine, can be employed in this mapping.

This paper is structured as follows. In the next chapter, we discuss the relations between this work and the literature. Then the implementation of the game controller is depicted. After that, two different mapping algorithms are implemented for a fighting game. The experiments show the quality of the control and people's attitudes towards the system. In the last chapter, we conclude and present the outlook for future work.

\section{RELATED WORK}

Ubiquitous computing, first proposed by Mark Weiser in 1988 [8], is receiving more and more attention in the domain of Human Computer Interactions(HCI). The main reason is that there are many embedded systems coming into our daily lives, which foster new ideas in HCI. Many works in HCI can be described as the replacement of the two interfaces: one is from human to computer, which are a mouse and a keyboard; another is from computer to human, which is a display. Instead, physical interfaces are developed, which are supposed to work in a way that the computer in a system is totally hidden behind these interfaces [3]. The end users of such a system cannot aware that a computer is being operated.

In some applications, the computers need to be directly observed and operated. The subject of this paper - digital games - can be classified into this kind of application. The reason is that the display of a digital game, e.g. the game of a simulated war, is actually a highlight of the system. For this kind of application, an important idea in the direction of HCI was proposed by Ishii and Ullmer in 1997: the operations of the virtual objects in the digital world are achieved in a "touchable" way, which is the so-called Tangible Bits [5]. To develop a game controller, researchers normally focus on 
how to create a physical interface to control the game, while the display is left untouched. Headon and Curwen developed a ubiquitous game controller in 2002 [6]. Sensors are installed under the floor so that the player's movements on the floor can be transferred to control a digital game.

Varieties of devices (sensors) have been used in game controllers in the past number of years. For example, RFIDSystem can be used as an input of a game [1], as well as voice [4]. The VoodooIO gaming kit [7] consists of configurable press-buttons. In this work, there are multiple sensors working together in the environment, which is the idea of ubiquitous computing [8]. Our game controller has a similar distributed architecture to [6] and [7]. A set of devices are integrated into a single controller, which is very different from the ones employed only one input method $[1,4]$. LMS is used for observing the surroundings. To our knowledge, the approach in this paper has three highlights: the game controller is based on a series of advance technologies which are real-time and require no physical contact for the control; the mapping module in the framework can significantly change a game, which makes a game, rather than easy and comfortable, exhausting for the players; the experiments show statistics on people's attitudes towards the mapping and the developed game controller.

\section{GAME CONTROLLER}

\subsection{System Architecture}

The system architecture is shown in Figure 1. There are three modules in the system: sensor module, mapping module, and game control module. The sensor module are distributed in several computers, which provides a flexible way to place the sensors at the proper places and to add computers for more computational power. A local area network (Ethernet) is established for the communication. The communication between two processes is achieved via a "socket". It takes less than $10 \mathrm{~ms}$ on sending and receiving a message.

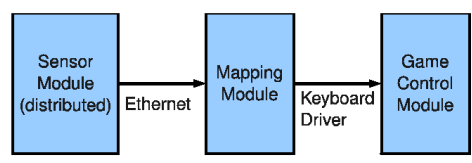

Figure 1: System Architecture

The mapping module is a must for the system. Most commercial games assume that they are controlled by pressing buttons (and) or a mouse, which should be done with ease by hand. In many cases, the skill of playing a game is to press the right buttons at the right times. When the game is controlled by an activity, the tasks are totally changed. Jumping and running from one place to another need time spans and efforts which are very different from pressing buttons. A game, designed for hands, can hardly be controlled by an activity. The main functionality of the mapping module is to bridge the gap between using "hands" and using an "activity" to control. It also provides a way to configure the game behaviors. The arrow between the mapping and the game control modules is implemented by hooking the keyboard events in the operating system.

All the components of the controller can be put into a mid- size suitcase, although the valid acting area of the controller can be as big as a room. The controller does not have many requirements of the environments. For example, it does not require a critical lighting condition as a normal camera does. It can be setup in short in a any typical room, or even outside. One shortcoming of the system is that its price is not yet ready for market.

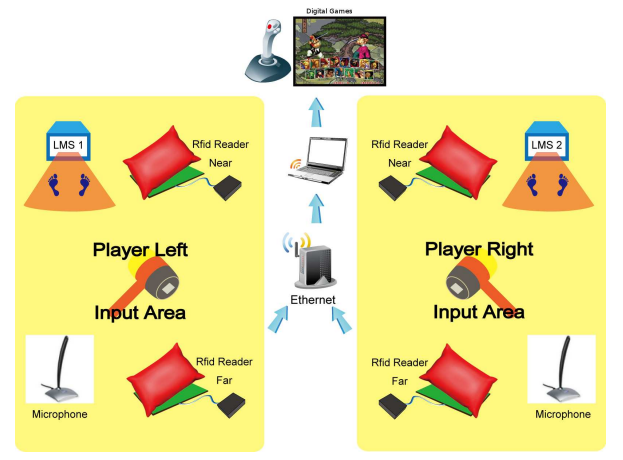

Figure 2: System Components

Figure 2 shows the system components. For each player, we use one LMS, one microphone, and two RFID-readers which can drive the player to walk or to run in between. Two sets of the devices make it possible for a human versus human game.

\subsection{Observation of the Legs}

Laser Measurement System (LMS) is a fast and accurate device to observe the surroundings. It emits a fan-shape laser beam. The measurements are a series of points, each with a distance and angle to the laser start point. $L M S 400^{4}$ is chosen to observe the legs' actions. It has a scan-frequency of about $350 \mathrm{~Hz}$; the laser open is $70^{\circ}$; the valid range of the measurements is from $700 \mathrm{~mm}$ to $3000 \mathrm{~mm}$ with an accuracy of $\pm 3 \mathrm{~mm}$. The LMS is set up on the ground in such a way that the laser beam projects on the lower part of the calf, as shown in Figure 3(a). When the player stands still, LMS can observe two round objects in its view.

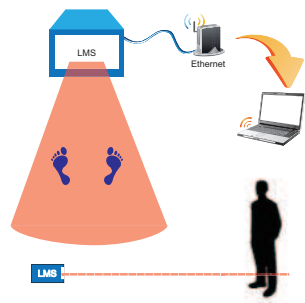

(a) LMS System

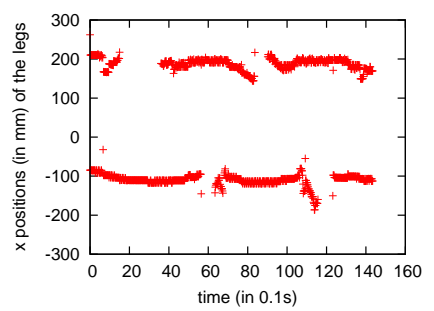

(b) Walking
Figure 3: Observing the Actions of the Legs

In order to detect the legs, an algorithm is implemented to trace the position of each leg. Figure 3(b) shows these data: $\mathrm{x}$ axis is the time, $\mathrm{y}$ axis is the $\mathrm{x}$ position of the legs; the observed player was walking. One leg is raised after another when a person is walking, and the LMS cannot observe a leg

${ }^{4} \mathrm{LMS}-400$ is a product of SICK AG 
if it raised higher than the laser beam. We can see in the data that the player walked for four steps. The sensor data is noisy, thus the recognition is implemented using a moving window.

\subsection{RFID-Tags and Voice as the Inputs}

Radio Frequency Identification (RFID) is a technology by which an ID can be read without any physical touch. It can identify many IDs. If the IDs are combined with other objects, e.g. the left hand and the right hand, the RFID system can recognize much more information than a press button. We chose an RFID reader and tags with a reading frequency of more than $100 \mathrm{~Hz}$, and a range of $150 \mathrm{~mm}$. Two RFID readers are used for each player, who has two toys with the tags. The readers are placed at the two ends of the active area, so that reading a tag at one reader and then the same tag at the other is an activity of walking (running) through the area.

Recognizing the content of a voice is a difficult task. To simplify the task, we use only the volume of the voice to control a game. If the output of the microphone is only zero (the background noises) and one (shouting at the microphone), a single threshold can lead to accurate and robust performance. As an input, a shout only needs to be loud enough. It also adds funny effects. Shouting during a game causes a lot of laughter for both the players and the spectators.

\section{MAPPING METHODS}

We chose a fighting game (Samurai $I I^{5}$ ) for several reasons. It needs a complex control sequence. Board games such as "FreeCell" and "Chess" are much less challenging. In addition, human versus human mode makes the game more exciting. The tasks of the game are quite simple: attacking the opponent as much as possible, while defending against being attacked. The character is controlled by three direction keys, and three action keys. In addition, each character has three or four advance actions, e.g. a sequence of right, down, left, boxing, and jumping will lead to a rush slash. The advance actions require more skills in the control, and in most cases they decide the results of a game.

In our system, the game is controlled in three distinguished modes. "Joystick" is the classic way to control a fighting game. This mode is regarded as a baseline for the estimation of the others. "Direct mapping" is an intuitive and natural way to map the activities of the player to the actions of the virtual character in the game. "Indirect mapping" mode mentions the activities of the player, in which the game is significantly simplified.

In the "direct mapping" mode, the human actions are directly mapped to a game action. The mapping is designed as intuitive and easy as possible. For example, the jump of the player is mapped to the jump of a virtual character. This is even more intuitive than "pressing a jump button". We also choose the toys similar to the weapons in the game. RFID tags are attached on an inflatable hammer and a rubber sword. Although the player should be much more active than when they are in the "joystick" mode, the "direct mapping" mode keeps most highlights of the game. Most basic

\footnotetext{
${ }^{5}$ Samurai II is a product of Neo Geo
}

and advance actions available in the game are still possible by using the game controller. The mapping of the advance actions is designed to be as close to the real movement as possible. For instance, if the player inputs using a sword, and then jumps, the game character will do the most similar action: a rising slash. The main disadvantage of the "direct mapping" is that it delays a bit from the time the player starts the action sequence to when the virtual character reacts to the command, especially when there is a long sequence of actions that need to be performed.

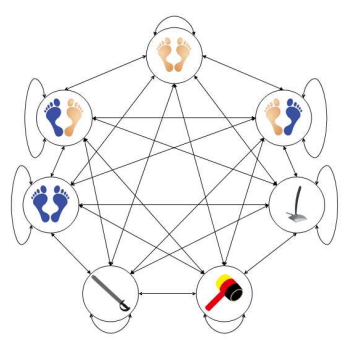

Figure 4: A Full-Connected Finite State Machine

In "indirect mapping" mode, we use a finite state machine to control the game. Figure 4 shows an example of such a state machine. Each node in the graph is the input of a device. The activities of the player can be mapped to a sequence of states and transitions. The activities of a player can thus be modeled and expressed in an indirect way. Considering the chosen fighting game, we configured the state machine as follows. Each transition is weighted by the required energy from one node to another. The weights are accumulated in the state machine - for example, the jump of a player can be mapped to a sequence of observations: two legs are observed, no leg is observed, and two legs are observed. The sequence is scored as 10. Walking activity can be recognized in a similar way, which is scored as 5 . The accumulated energy can be released to the virtual character of the game by a special node, e.g. sword. The "indirect mapping" significantly changes the game. The only skill a player needs is to release the energy at a proper time, which makes the game very easy for the beginners. The player would feel quite exhausted after only two or three rounds.

\section{EXPERIMENTS}

The experiments were done in the following scenario. First, the participants play the game with a joystick, so that they get to know the basic operations and the advance skills of the game. Then, they are invited to play against each other using the "direct mapping" mode. Before they start to play, we give a short introduction to the mapping. The instructions are introduced and demonstrated one by one. Then, they can play by themselves while looking at the instruction page. After that, the "indirect mapping" mode is introduced in brief and they are asked to play using this mode. Finally, they evaluate and compare three control modes by completing a questionnaire.

The evaluation takes the form of scores: minus is negative; zero is neutral, and plus is positive. Questions $1-4$ are used to evaluate the quality of the control. They cover four aspects: easy to use, reactive, accurate, and fully-controlled. 
Question 5 is designed to evaluate whether or not the game controller is novel for the participants. Question 6 estimates how healthy a digital game could be. Question 7 ask the participants to rate the three control modes overall. Finally, we got 31 participants, 28 of which completed the questionnaire.

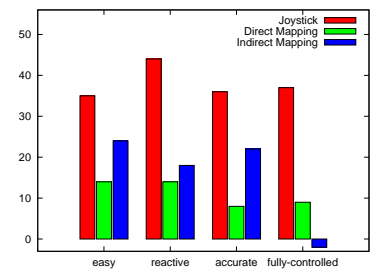

(a) Quality

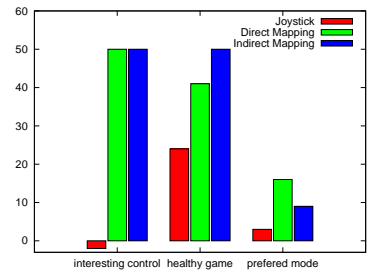

(b) Attitude
Figure 5: Statistics of the Returned Questionnaires

Figure 5(a) shows the sum of the scores of the questions $1-4$. The joystick has a better control quality over all of the four aspects. There are mainly two reasons for this result. On the one hand, a joystick is a classic control device, thus most people are good at using it. On the other hand, the game was originally designed for running in a "game machine", which has a control interface very similar to a joystick. The "direct mapping" turns out to be more difficult, less reactive, and less accurate to be controlled than the "indirect mapping" in the experiments. "Direct mapping" is more difficult because the mapping contains an instruction list, which is new for all the participants; they have to learn these instructions during the games, whereas, the "indirect mapping" has an extra GUI and the state machine calculated whatever the player did as a certain amount of energy, which is quite reactive. The final trigger to release action is very easy. Therefore, the control using "indirect mapping" can be more reactive and accurate. "Indirect mapping" was evaluated as negative in the fully-controlled because the game actually cannot be fully-controlled in this mode.

Figure 5(b) shows the attitudes of the participants towards the three control modes. The joystick was not interesting for the participants. The other two modes were equally novel. Many people gave surprisingly positive feedback towards the joystick on healthy game question. A reasonable explanation is that the evaluation process was quite short in time; participants were not aware that moving only their fingers and gazing at the screen for long periods of time is somehow unhealthy. Compared to the other modes, "indirect mapping" induces movement the most, so it was estimated as the most healthy game. "Direct mapping" was also significantly better than the joystick. The participants were asked to exclusively choose a favorite mode in the last question. 16 people preferred the "direct mapping", even if the control quality of the mode is the worst one. 9 people preferred "indirect mapping"; only 3 people remained with the joystick. A possible explanation is that "direct mapping" not only introduces much more movements, but also keeps the most interesting feature of the original game. "Indirect mapping" is also interesting, but it requires much less skill.

\section{CONCLUSION AND OUTLOOK}

In this paper, A game controller is developed by using three distinctive measurement methods, which require no physi- cal touch. We chose a fighting game as a case study. Two different mapping modes were implemented. People participated in the evaluation of the game system. The experiment's statistics show that mapping module can significantly change the game. The game controller was pretty interesting for the participants. It achieved a fairly good evaluation in the quality of the control. The participants preferred to use the controller, although it did not have a control quality as high as that of a joystick.

Instead of making the game control easier and more comfortable, we are working towards making the control be taxing or even exhausting for the players. The experiments show that people actually accept this idea quite well. Most of the participants preferred to play the game using the developed game controller, rather than a classic joystick. It is possible that some highlights of a game are limited by the controller, which is a shortcoming of the system. The mapping method needs to be chosen very carefully.

In the future, other measurement techniques can be integrated into the controller. For example, if there is an eye tracking camera by which the computer knows where the player is looking, the operations of a mouse become feasible for the game controller. The activities of players could be studied. In this work, we did not actually pay much attention to this. It would be wonderful if an interesting sport could be combined with a digital game.

\section{REFERENCES}

[1] J. Bohn. The smart jigsaw puzzle assistant: Using rfid technology for building augmented real-world games. Workshop on Gaming Applications in Pervasive Computing Environments at Pervasive 2004, Vienna, Austria, Apr. 2004.

[2] A. Craig, B. Leonard, D. Edward, H. Rowell, J. James, L. Daniel, M. Neil, and Wartellaellen. Voilent video game effects on children and adolescents. 2003.

[3] S. Greenberg and C. Fitchett. Phidgets: easy development of physical interfaces through physical widgets. In UIST '01: Proceedings of the 14th annual ACM symposium on User interface software and technology, pages 209-218. ACM Press, 2001.

[4] P. Hämäläinen and J. Höysniemi. A computer vision and hearing based user interface for a computer game for children. In Proceedings of 7th ERCIM Workshop, "User Interfaces for All", Oct. 2002.

[5] H. Ishii and B. Ullmer. Tangible bits: towards seamless interfaces between people, bits and atoms. In $\mathrm{CHI}$ '97: Proceedings of the SIGCHI conference on Human factors in computing systems, pages 234-241, New York, NY, USA, 1997. ACM Press.

[6] H. Robert and C. Rupert. Movement awareness for ubiquitous game control. Personal Ubiquitous Comput., 6(5-6):407-415, 2002.

[7] N. VILLAR, K. M. GILLEADE, D. RAMDUNYELLIS, and H. GELLERSEN. The voodooio gaming kit: a real-time adaptable gaming controller. Comput. Entertain., 5(3):7, 2007.

[8] M. Weiser. The computer for the 21st century. Scientific American, 02/1991 1991. 